\title{
Consensus Report on Gastric Emptying: What's Needed to Prevent Tarnishing a Gold Standard?
}

$\mathbf{T}$ the nuclear medicine community a new consensus report that establishes a standardized methodology for performing gastric-emptying studies. This consensus was achieved after a series of meetings jointly sponsored by the SNM Gastroenterology Council and the American Neurogastroenterology and Motility Society. The report has already appeared in press in the American Journal of Gastroenterology and appears this month in the Journal of Nuclear Medicine Technology $(1,2)$.

This consensus report is an example of the cooperation that needs to be fostered between similarly oriented medical professional groups. As pay-for-performance programs become a reality, government and other thirdparty payers will be looking for professional societies to set standards for the performance and interpretation of diagnostic studies. If such standards are the consensus of experts from multiple medical professional groups, they will more quickly be recognized and established as standards for patient care.

Although gastric-emptying scintigraphy in most clinical practices is the gold standard for measuring gastric motility, there has been concern that it will lose its role to other competing modalities because of a lack of standardization (3).

As stated in the report, currently "there is a lack of standardization of the test, including differences in meals used, patient positioning, frequency, and duration of imaging. There are differences in the quantitative data reported, e.g., half-time of emptying, rate of emptying (percent per minute), or the percent retention or emptying at different time points during the study. Normal values often have not been established for some of the protocols used, and the performance characteristics of the test with the specified meal may not have been established or published. Lack of standardization limits the clinical utility of the test, and presents

Received Dec. 12, 2007; revision accepted Dec. 14, 2007.

For correspondence or reprints contact: Alan $\mathrm{H}$. Maurer, MD, Temple University Hospital and School of Medicine, 3401 N. Broad St., Broad and Ontario Streets, Philadelphia, PA 19140.

E-mail: amaurer@temple.edu

COPYRIGHT ( 2008 by the Society of Nuclear Medicine, Inc.

DOI: 10.2967/jnumed.106.037507

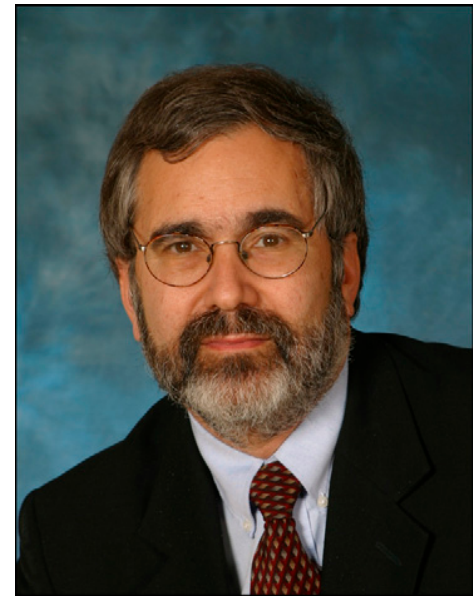

problems for patients and their physicians as the latter try to interpret study results from other institutions" (1).

To address these issues, the consensus group worked to develop recommendations on how to perform a solid-meal gastric-emptying test for routine clinical use "using readily available technology and normative data, which can provide clinicians with standardized results." The report "addresses those aspects that the multidisciplinary group considers were in the greatest need of immediate standardizationthe meal, the frequency of imaging, the duration of the test, and the normative data."

The consensus statement recommends use of a low-fat, egg-white meal with images acquired at $0,1,2$, and $4 \mathrm{~h}$ after meal ingestion based on a large multicenter study (4). In a series of appendices, the report includes additional recommendations on patient preparation, meal preparation, image acquisition and analysis, reporting, and patient instructions, as well as a sample patient questionnaire used to acquire clinical information important to those interpreting and receiving the results of a gastric-emptying study.

The report also recognizes our increasing appreciation of the complexity of gastric motility and the ongoing investigations of how scintigraphy can promote our understanding of the physiology of gastric digestion and how this relates to patients' symptoms (5). The report includes a list of issues that require further clarification, including optimization of imaging time points, need for normative data on other non-egg-solid meals, glycemic control in di- abetic patients, the value of monitoring symptoms during the study, a scale to assess the severity of delayed gastric emptying, the need for postoperative reference data, the clinical role of analyzing fundal and antral gastric function, other methods of quantitation (curve fitting, lag phase, total abdominal counts), and the need for industry to provide standardized software for analysis.

This report has been reviewed and approved by the SNM Practice Standards Committee and Procedure Guidelines Committee. It is anticipated that these recommendations will become incorporated into new SNM procedure guidelines. The Gastrointestinal Council of the SNM believes the publication of this report is an important example demonstrating how the SNM can partner with its referring physicians to understand their needs and provide them with accurate and consistent findings. The SNM is now proceeding to develop additional standards for gastrointestinal motility studies in the areas of cholecystokinin cholescintigraphy and esophageal, small-bowel, and large-bowel transit. The SNM urges all those performing gastric-emptying studies to quickly adopt these new standards so we can achieve consistency and reliable results for our patients and referring physicians.

\section{REFERENCES}

1. Abell TL, Camilleri M, Donohoe K, et al. Consensus recommendations for gastric emptying scintigraphy: a joint report of the American Neurogastroenterology and Motility Society and the Society of Nuclear Medicine. Am J Gastroenterol. 2007;102:1-11.

2. Abell TL, Camilleri M, Donohoe $\mathrm{K}$, et al. Consensus recommendations for gastric emptying scintigraphy: a joint report of the American Neurogastroenterology and Motility Society and the Society of Nuclear Medicine. J Nucl Med Technol. 2008;36:44-54

3. Maurer A. Can we prevent tarnishing a gold standard? [editorial]. Semin Nucl Med. 1995;25:288.

4. Tougas G, Eaker E, Abell T, et al. Assessment of gastric emptying using a low-fat meal: establishment of international control values. Am J Gastroenterol. 2000;95:1456-1462.

5. Maurer AH, Parkman H. Update on gastrointestinal scintigraphy. Semin Nucl Med. 2006;36:110-118.

Alan Maurer

Temple University Hospital and School of Medicine

Philadelphia, Pennsylvania 